Introduction: Immune responses within the tumor depend on the ability of leukocytes to migrate from peripheral circulation into the local microenvironment. This process is controlled by mechanisms that guide leukocytes to the side of inflammation, allowing them to cross vascular endothelial barrier. Monocytes/macrophages are the predominant population of leukocyte infiltrate of many tumors, including, gastric cancer. However, to date mechanisms that control monocyte trafficking to the side of tumor growth are not fully elucidated.

Aim of the study: It this study we aimed to evaluate transmigratory potential of peripheral blood monocytes from gastric cancer patients.

Material and methods: By using multicolor flow cytometry we assessed expression of $\beta 1$ - and $\beta 2$-integrins on peripheral blood monocytes from gastric cancer patients.

Results: We found increased frequencies of VLA-4 and VLA- 6 expressing monocytes and increased expression of analyzed $\beta 2$-integrins in gastric cancer patients when compared to age matched controls.

Conclusions: In summary, this study revealed that gastric cancer increases transmigratory potential of peripheral blood monocytes.

Key words: gastric cancer, monocytes, $\beta 2$-integrins, VLA-4, VLA6.

Contemp Oncol (Pozn) 2018; 22 (1A): 33-37 DOI: https://doi.org/10.5114/wo.2018.73881

\section{Gastric cancer increases transmigratory potential of peripheral blood monocytes by upregulation of $\beta 1$ - and $\beta 2$-integrins}

Andrzej Eljaszewicz ${ }^{1}$, Michal Jankowski' ${ }^{2,3}$, Malgorzata Wiese-Szadkowska ${ }^{4}$, Lidia Gackowska ${ }^{4}$, Jacek Michalkiewicz ${ }^{4}$, Wojciech Zegarski ${ }^{2,3}$, Marcin Moniuszko ${ }^{1}$

${ }^{1}$ Department of Regenerative Medicine and Immune Regulation, Medical University of Bialystok, Poland

${ }^{2}$ Department of Surgical Oncology, Collegium Medicum in Bydgoszcz, Nicolaus

Copernicus University of Torun, Poland

${ }^{3}$ Oncology Centre - Prof Franciszek Lukaszczyk Memorial Hospital, Bydgoszcz, Poland

${ }^{4}$ Department of Immunology, Faculty of Pharmacy, Collegium Medicum in Bydgoszcz, Nicolaus Copernicus University in Torun, Poland

\section{Introduction}

Macrophages represent predominant component of leukocyte infiltrate in many tumors, including gastric cancer [1, 2]. Due to their pleiotropic biological activities they act as orchestrators of immune response within tumor. They may play pivotal role in tumor development acting as tumor suppressors (M1 phenotype - classically activated cells) or tumor supporters ( $M 2$ phenotype - alternatively activated cells) [1, 3]. Unfortunately, majority of macrophages within tumor microenvironment acquire the latter phenotype and are referred to as tumor associated macrophages (TAMs). Macrophage polarization depends on the immune modulatory properties of tumor and stroma cells, that can interact locally within tumor tissue or affect peripheral precursors (monocytes) [4].

Recruitment of monocytes into tumor microenvironment is a hallmark of cancer development and progression [5, 6]. Notably, migration of peripheral blood cells to the side of tumor growth is controlled by different soluble factors, namely cytokines, chemokines, growth factors and metabolites [7, 8]. On the other hand, vascular and epithelial junctions represent a barrier for leucocyte migration [9]. Interestingly, monocyte transmigration through vessel wall is possible due to the presence of membrane adhesion molecules, including proteins belonging to $\beta 1$ and $\beta 2$-integrin family [10]. Leukocyte adhesion molecules interact with their ligands expressed on cytokine activated endothelium and allows monocytes to avoid forces exerted by the rapid blood flow in vasculature. Consequently, monocytes start to roll along apical endothelial surface until complete immobilization and transmigration [11]. Unfortunately, to date the molecular, humoral and cellular mechanisms that control monocyte trafficking in cancer are not fully elucidated. Therefore, here we aimed to evaluate whether systemic activation of peripheral blood monocytes observed in gastric cancer patients increases transmigratory potential of these cells.

\section{Material and methods}

\section{Patients}

15 normal donors and 40 gastric cancer patients, succsesive qualified to stomach resection at Chair of Surgical Oncology, Prof. F. Lukaszczyk Memo- 
Table 1. Clinical characteristics of study population

$\begin{array}{lcc}\text { Parameter } & \text { Gastric cancer } & \text { Normal } \\ n & 40 & 17 \\ \text { Mean age (range) } & 62,79(30-86) & 58,35(49-68) \\ \text { Gender (female/male) } & 13 / 27 & 8 / 9 \\ \text { Stage according to AJCC (frequencies of all) } & \\ \text { Stage I } & 12(30 \%) & \\ \text { Stage II } & 2(5 \%) & \\ \text { Stage III } & 14(35 \%) & \\ \text { Stage IV } & 12(30 \%) & \end{array}$

AJCC - American Joint Committee on Cancer

rial Centre of Oncology in Bydgoszcz (Poland), were enrolled to the study (Table 1). None of the patients received chemotherapy and radiotherapy before or was subjected to surgery or blood transfusion for at least six month before blood acquisition. Furthermore, none of the patients showed any clinical or cellular sings on ongoing infection. Peripheral blood was collected upon the approval of the Bioethical Committee of the Collegium Medicum in Bydgoszcz. Each participant was familiarized with the objectives of the study and expressed written consent.

\section{Flow cytometry}

$100 \mu \mathrm{l}$ of fresh heparin-anticoagulated blood was stained with panel of mouse anti-human monoclonal antibodies (Table 2). Stain-then-lyse protocol was used as previously described [12]. Appropriate fluorescence-minus-one (FMO) and isotype controls were used for every staining for setting compensation and to assure correct gating. Samples were analyzed by using FACScan flow cytometer (Becton Dickinson) and at least 40000 events were collected. Next, collected data were analyzed by using FlowJo version 7.6.1. (TreeStar). Used gating strategy is presented on Fig. 1.

\section{Statistics}

Statistical analysis was carried out using GraphPad Prism 6 software (GraphPad Software). U Mann-Whitney test was used. The differences were considered statistically significant at $p<0.05$. The results are presented as median (interquartile range).

\section{Results}

Due to the constitutive expression of analyzed $\beta_{2}$-integrins on the surface of monocytes we analyzed their expression levels. First we found significant increase of CD11a and CD11b expression in gastric cancer patients when compared to normal donors. Additionally, we found no differences in CD11C and CD18 expression level. Next, we found that gastric cancer increase frequencies of CD49d ( $\alpha 4$ subunit of VLA-4 integrin) and CD49f ( $\alpha 6$ subunit of VLA- 6 integrin) expressing monocytes. Interestingly, we did not observed any differences in expression level of above mentioned molecules.

\section{Discussion}

Integrins are membrane glycoproteins controlling numerous physiological processes, including cell adhesion, chemotaxis, and phagocytosis [13]. $\beta 2$-integrins are heterodimeric receptor proteins consisting of $\alpha$ and $\beta$ subunits linked by sulphide bridges. All receptors shear common $\beta 2$-chain (CD18) and differ in $\alpha$ chain variants, namely $\alpha L-C D 11 a ; \alpha M-C D 11 b ; \alpha X-C D 11 c$. They are involved in direct adhesion of leukocytes to endothelial cells [14, 15]. Similarly, $\beta 1$-integrins shear common $\beta 1$ chain (CD29) and differ in $\alpha$ subunits. To date, at least 10 different $\alpha$ chains were discovered including CD49d and CD49f expressed on monocytes. In contrast to $\beta 2$-integrins, the latter represent a group of protein receptor responsible for cell interactions with extracellular matrix [13]. Here, we found that gastric cancer increase frequencies of both VLA-4 and VLA-6 expressing monocytes. Interestingly, Jin et al. showed that VLA-4 is playing leading role in monocyte transmigration to tumor microenvironment [16]. However, in some contrast to previous studies by Zhang et al., they found that this process occurred in the $\alpha M \beta 2$ (CD11b/CD18) integrin independent manner $[16,17]$. It seems, that $\beta 2$-integrins may play supportive role in monocyte transmigration process and observed upregulation of CD11a and CD11b expression is a consequence of monocyte activation and their inflammatory phenotype [18]. Notably, in our previous report we found that gastric cancer patients showed increased frequencies of inflammatory (activated) monocytes, namely intermediate (CD14++CD16+) and non-classical (CD14+CD16++) cells [12]. Interestingly, VLA-4 support not only transmigration of inflammatory monocytes but also

Table 2. Characteristics of monoclonal antibodies used in the study

$\begin{array}{lcccc}\text { Name } & \text { Clone } & \text { Isotype } & \text { Format } & \text { Manufacturer } \\ \text { CD14 } & \text { MoP9 } & \text { IgG2b } & \text { PerCP } & \text { FITC } \\ \text { CD49d (VLA-4 } \alpha \text { subunit) } & 9 F 10 & \text { IgG1 } & \text { FITC } & \text { Becton Dickinson Dickinson } \\ \text { CD49f (VLA-6 } \alpha \text { subunit) } & 1 . \text { BB.460 } & \text { IgG2b } & \text { FITC } & \text { Becton Dickinson } \\ \text { CD11a } & \text { G43-25B } & \text { IgG2a } & \text { PE } & \text { Santa Cruz Biotechnology } \\ \text { CD11b } & \text { I.BB.189 } & \text { IgG1 } & \text { Becton Dickinson } & \text { Becton Dickinson }\end{array}$

PerCp-peridinin chlorophyll protein complex; FITC - fluorescein isothiocyanate; PE - phycoerythrin 

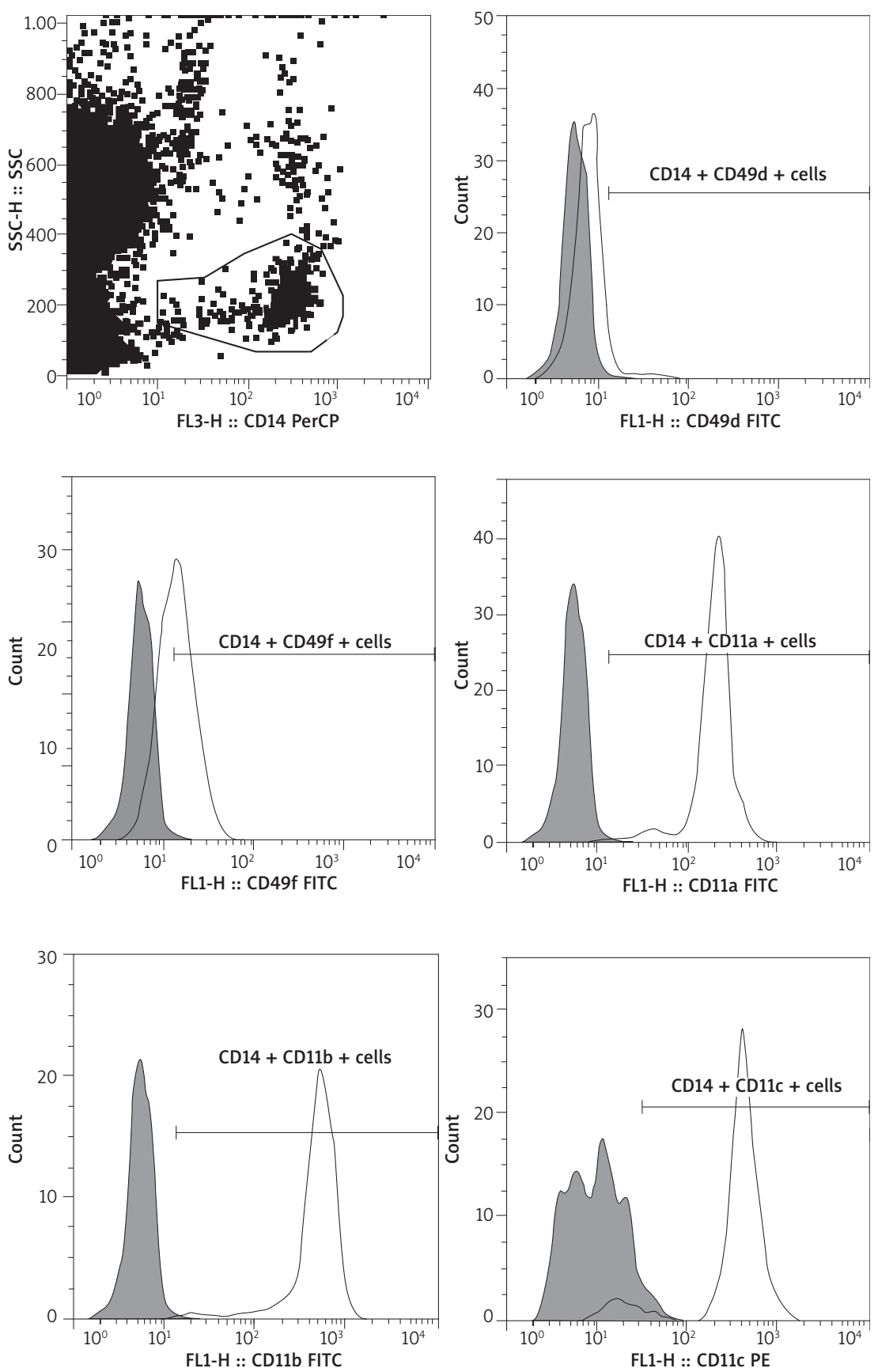

\section{References}

1. Eljaszewicz A, Wiese M, Helmin-Basa A, et al. Collaborating with the enemy: function of macrophages in the development of neoplastic disease. Mediators Inflamm 2013; 2013: 831387.

2. Eljaszewicz A, Gackowska L, Kubiszewska I, et al. Macrophage activity in tumour development. Contemp Oncol (Pozn) 2010; 14: 1-6.

3. Solinas G, Germano G, Mantovani A, Allavena P. Tumor-associated macrophages (TAM) as major players of the cancer-related inflammation. J Leukoc Biol 2009; 86: 1065-73.

4. Mantovani A, Sozzani S, Locati M, et al. Macrophage polarization: tumor-associated macrophages as a paradigm for polarized M2 mononuclear phagocytes. Trends Immunol 2002; 23: 549-55.

5. Baggiolini M. Chemokines and leukocyte traffic. Nature 1998; 392: 565-8.

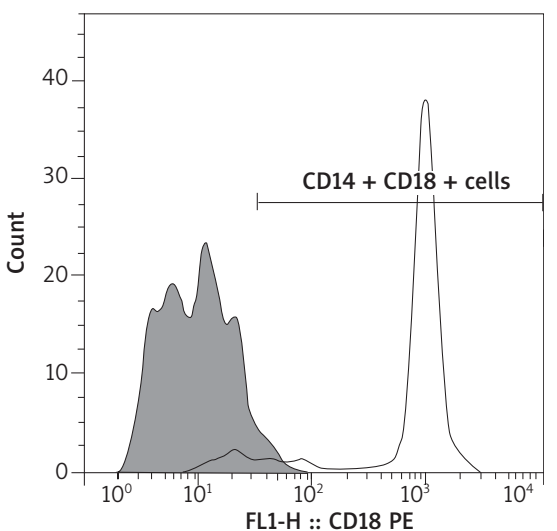

6. Schmid MC, Varner JA. Myeloid cell trafficking and tumor angiogenesis. Cancer Lett 2007; 250: 1-8.

7. Nagarsheth N, Wicha MS, Zou W. Chemokines in the cancer microenvironment and their relevance in cancer immunotherapy. Nat Rev Immunol 2017; 17: 55972.

Fig. 1. Gating strategy for monocytes. Representative FACS plots demonstrating gating strategy to evaluate CD49d (VLA-4 $\alpha$ subunit), CD49f, CD11a, CD11b, CD11c and CD18 expressing monocytes and fluorescence intensity of analyzed receptors

FL1-H :: CD18 PE 

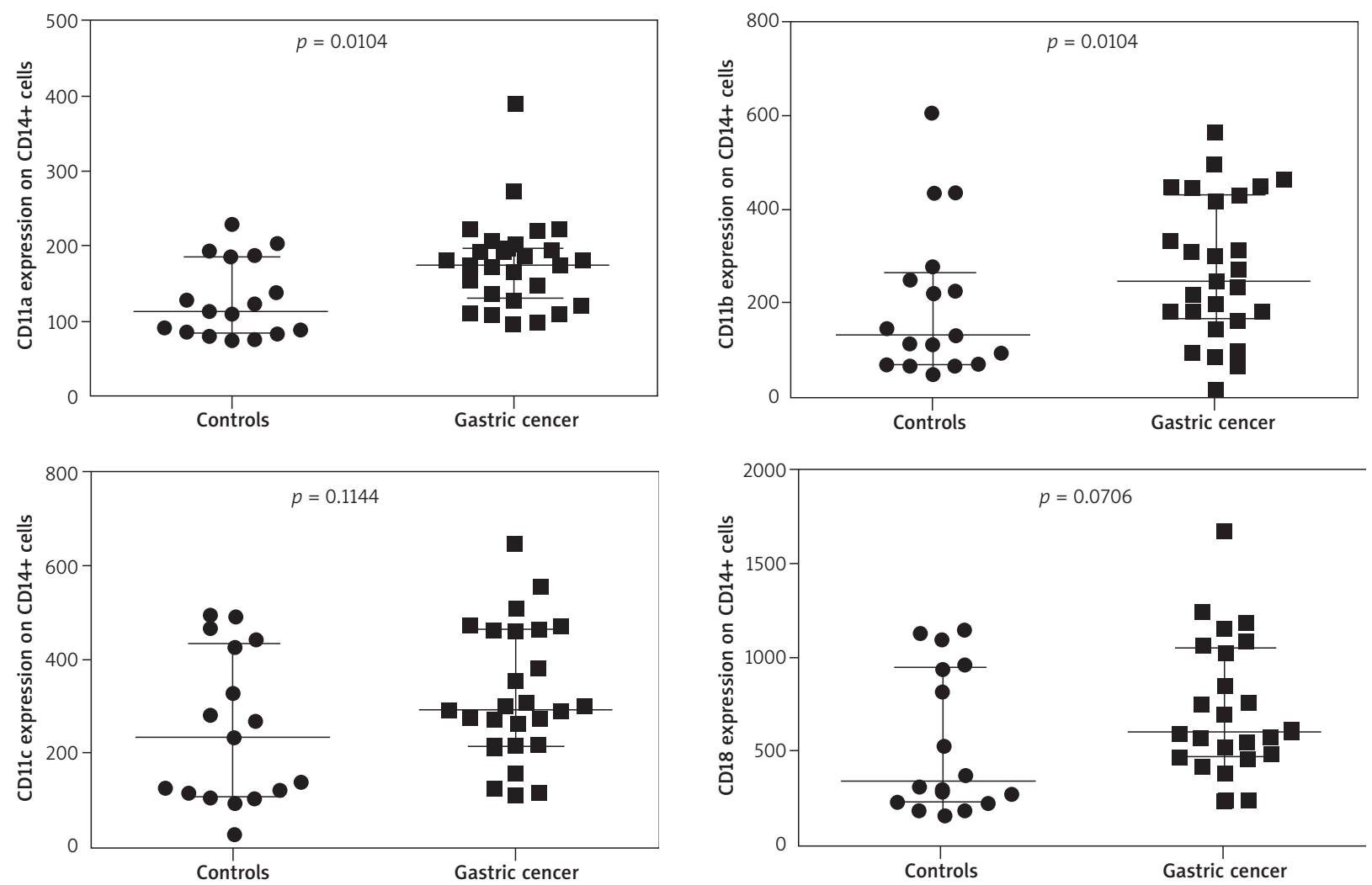

Fig. 2. $\beta 2$-integrin expression in peripheral blood monocytes. Summary of analyses of (A) CD11a, (B) CD11b, (C) CD11c and (D) CD18 expression on CD14+ cells. Data are presented as median and interquartile range
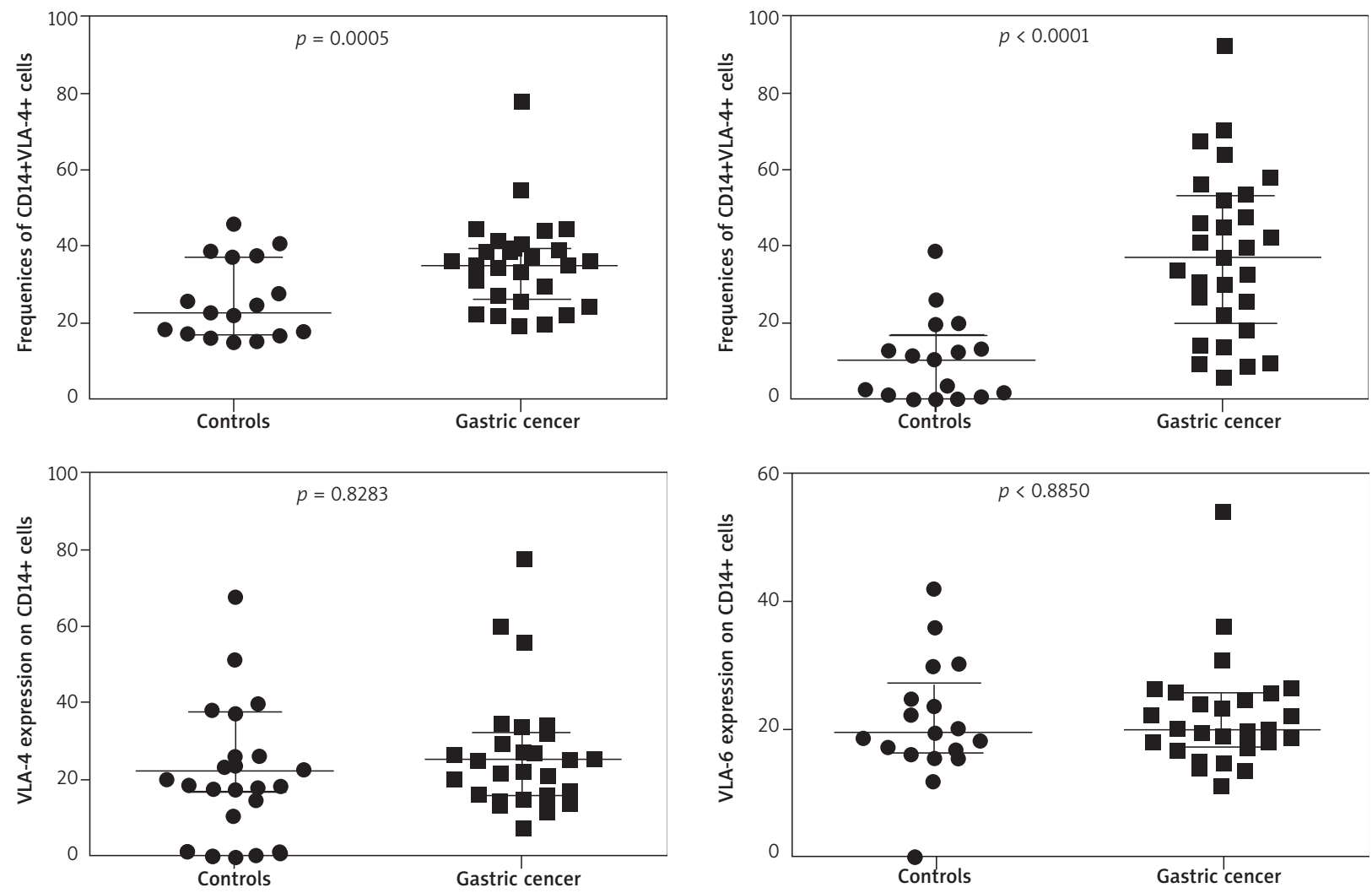

Fig. 3. VLA-4 and VLA-6 expression in peripheral blood monocytes. Summary of analyses of (A) VLA-4 and (B) VLA-6 expressing monocytes and (C) VLA-4 and (D) VLA-6 expression on CD14+ cells. Data are presented as median and interquartile range 
10. Vestweber D. How leukocytes cross the vascular endothelium. Nat Rev Immunol 2015; 15: 692-704.

11. Gerhardt T, Ley K. Monocyte trafficking across the vessel wall. Cardiovasc Res 2015; 107: 321-30.

12. Eljaszewicz A, Jankowski M, Gackowska L, et al. Gastric cancer increase the percentage of intermediate (CD14++CD16+) and nonclassical (CD14+CD16+) monocytes. Centr Eur I Immunol 2012; 37: 355-61.

13. Hynes RO. Integrins: versatility, modulation, and signaling in cell adhesion. Cell 1992; 69: 11-25.

14. Walzog B, Weinmann P, Jeblonski F, et al. A role for beta(2) integrins (CD11/CD18) in the regulation of cytokine gene expression of polymorphonuclear neutrophils during the inflammatory response. FASEB J 1999; 13: 1855-65.

15. Kourtzelis I, Mitroulis I, von Renesse J, et al. From leukocyte recruitment to resolution of inflammation: the cardinal role of integrins. J Leukoc Biol 2017; 102: 677-83.

16. Jin H, Su J, Garmy-Susini B, et al. Integrin alpha4beta1 promotes monocyte trafficking and angiogenesis in tumors. Cancer Res 2006; 66: 2146-52.

17. Zhang L, Yoshimura T, Graves DT. Antibody to Mac-1 or monocyte chemoattractant protein-1 inhibits monocyte recruitment and promotes tumor growth. J Immunol 1997; 158: 4855-61.

18. Chuluyan HE, Issekutz AC. VLA-4 integrin can mediate CD11/ CD18-independent transendothelial migration of human monocytes. J Clin Invest 1993; 92: 2768-77.

19. Joshi S, Singh AR, Zulcic M, et al. Rac2 controls tumor growth, metastasis and M1-M2 macrophage differentiation in vivo. PLoS One 2014; 9: e95893.

20. Bouvard C, Segaoula Z, De Arcangelis A, et al. Tie2-dependent deletion of $\alpha 6$ integrin subunit in mice reduces tumor growth and angiogenesis. Int J Oncol 2014; 45: 2058-64.

\section{Address for correspondence}

\section{Andrzej Eljaszewicz}

Department of Regenerative Medicine and Immune Regulation

Medical University of Bialystok

Waszyngtona 13

15-269 Bialystok, Poland

e-mail: andrzej.eljaszewicz@umb.edu.pl

\section{Marcin Moniuszko}

Department of Regenerative Medicine and Immune Regulation

Medical University of Bialystok

Waszyngtona 13

15-269 Bialystok, Poland

e-mail: Marcin.Moniuszko@umb.edu.pl 